\title{
La Planificación Estratégica como instrumento de gestión pública en el gobierno local: Análisis de caso
}

\section{Strategic Planning as a management tool in local government}

\author{
Ángel Iglesias Alonso ${ }^{1}$ \\ Universidad Rey Juan Carlos
}

Recibido el 20 de abril de 2009, aceptado el 12 de enero de 2010.

$\mathrm{N}^{\circ}$ de clasificación JEL: H70: State and Local Government

DOI: $10.5295 /$ cdg.100156ai

\section{Resumen:}

Los responsables políticos y los gestores de los gobiernos locales de las ciudades se enfrentan con un problema recurrente: cómo atender, con recursos cada vez más escasos, las demandas de sus ciudadanos y a los actores con interés en su territorio, en términos tanto de eficacia en la prestación de servicios como de profundización democrática. Utilizando una metodología cualitativa, el objetivo de este trabajo es identificar hasta que punto un proceso de planificación estratégica pública sirve para compatibilizar las demandas de democracia y eficacia en el gobierno y administración pública local, involucrando a los ciudadanos y a los actores económicos privados. Este estudio de caso demuestra esa posibilidad aunque se hacen necesarias investigaciones comparativas adicionales.

\section{Palabras clave:}

Gobernanza local, planificación estratégica de ciudades, participación ciudadana, modernización administrativa.

\begin{abstract}
:
Local councillors and public managers have to face a recurrent problem: with scarce resources they have to be responsive to the citizen's demands and other actors in the local arena, combining the delivery of efficient local services with democratic quality. Using qualitative data, this article analyses a process of strategic planning in a local government to ascertain to what extent such a process contributes to conciliate the demands of more local democracy and better provision of public services with the participation of both citizens and local economic actors. Evidence provided by this case is positive although more comparative research is needed.
\end{abstract}

Key words:

Local governance, city strategic planning, citizen's participation, public administration modernization.

${ }^{1}$ Universidad Rey Juan Carlos, $\mathrm{P}^{\mathrm{o}}$ de los Artilleros, s/n, 28032 Madrid. angel.iglesias@urjc.es 


\section{INTRODUCCIÓN}

A lo largo de las dos últimas décadas los gobiernos locales de los países de la OCDE han desarrollado Planes Estratégicos como instrumentos de gestión pública innovadora. Por Planificación Estratégica en la administración local se entiende el proceso de identificar las fortalezas, debilidades, oportunidades y retos de la organización administrativa municipal con objeto de definir su misión y objetivos, identificar a sus actores críticos (stakeholders) y configurar estrategias que mejoren la eficiencia de la organización (BRYSON, 2004; MINTZBERG, LAMPEL, QUINN Y GHOSAL, 2003.) Así planteada la Planificación Estratégica supone dos cosas: 1) mediante la introducción del pensamiento estratégico los gobiernos locales miran a su entorno e interactúan con él en busca de mejorar la competitividad de la ciudad y la calidad de los servicios públicos locales (VILLORIA, 1997; BELTRAN, 2000) y, en definitiva, el bienestar de sus vecinos; b) al mismo tiempo se persigue un funcionamiento de la organización municipal que a partir del ejercicio del liderazgo político se aleje del tradicional modelo burocrático (STOKER, 2008). En efecto, más allá de la propia estructura organizativa municipal, la participación en el proceso de planificación de actores críticos externos es susceptible de traer consigo la introducción de innovaciones que supongan cambios sustanciales en la estructura y el funcionamiento de la Administración Local (POISTER Y STREIB, 2005). En otro orden de cosas, la complejidad de los problemas locales exige transferencias de información y, a este respecto, la Planificación Estratégica de ciudades permite conceptualizar una visión del territorio, formular objetivos para su consecución y elaborar proyectos para la consecución de los objetivos. Es, en efecto, un instrumento de la gestión contextual para la gobernanza local ${ }^{1}$, es decir, de la creación de un proceso en el que todos los actores críticos de la ciudad (con sus valores, expectativas y experiencias), respetando su autonomía, se ligan interactivamente por medio de relaciones de reconocimiento mutuo y que posibilitan el entendimiento intersubjetivo y el compromiso. Ello exige a la administración municipal la coordinación horizontal a través de la creación de capacidades de planificación para la consecución de objetivos generales, además de la cooperación, interactividad e interpenetración de cargos electos, directivos y técnicos municipales (DENTERS y ROSE, 2005; AGRANOFF, 2007).

En España, en el ámbito territorial, existen actualmente alrededor de unos cien Planes Estratégicos. La ciudad pionera fue Barcelona que inició un Plan Estratégico aprovechando su nombramiento como ciudad olímpica. Si bien más de la mitad son Planes estratégicos

\footnotetext{
${ }^{1}$ El concepto de gobernanza local emerge como un concepto alternativo para analizar el modo de gobierno, organización y acción pública del gobierno local. Su utilidad estriba en que proporciona un marco analítico para la comprensión de los procesos de gobierno y gestión local que no se identifica sólo con las instituciones políticoadministrativas, pues este concepto se fundamenta en la existencia de redes funcionales conformadas por actores públicos y privados que, aunque con intereses conflictivos, a priori, se ponen en situación de cooperación para la consecución de sus objetivos. Ello significa que los centros de decisión no se residencian con exclusividad en el gobierno y administración local, sino en redes complejas que configuran entramados de toma de decisiones. En el contexto de estas redes, el gobierno local aparece como un actor que tiene que legitimarse liderando procesos y estableciendo sistemas de dación de cuentas. La gobernanza local hace énfasis en la interacción horizontal entre actores encuadrados en estructuras económicas, sociales, culturales y asociaciones ciudadanas con fines o valores diversos pero con intereses convergentes en el territorio de la ciudad. En este contexto, las prácticas de Planificación Estratégica son una derivada o instrumento del modelo de gobernanza que, al mismo tiempo, lo configuran e influyen.
} 
de ciudad (ARTHUR ANDERSEN, 1989; FERNANDEZ GÜELL, 1997, PASCUAL i ESTEVE, 2002) esta cifra incluye planes estratégicos de alcance metropolitano, comarcal o provincial, insular e, incluso, transfronterizos. La mayoría de ellos están siendo promovidos y liderados desde los poderes públicos, pero muchos de ellos se han quedado en la fase de diseño y no han llegado a ser implantados. De manera que en los debates sobre la gestión del crecimiento y desarrollo de las ciudades para hacer frente a los retos de la globalización se viene proponiendo, desde diversos campos, la Planificación Estratégica como instrumento de gobernanza local en red (BRUGUÉ, GOMÁ y SUBIRATS, 2002; GONZÁLEZ GÓMEZ y ORTEGA ALMÓN, 2003; LARREA ARANGUREN, 2003). Ahora bien, no existe aún un estudio sistemático de la realidad de buena parte de los esfuerzos en este ámbito, pero diversos autores vienen señalando como causas de los fracasos la falta de liderazgo en la implantación de las estrategias, las resistencias de la organización burocrática a interactuar con otros actores en lo que supone recortar el margen de acción de los administradores públicos o la dificultad para tomar decisiones por los vetos que puedan imponer actores externos a la organización municipal (FRENCH y FOLZ, 2004; PORTH, 2003; WHEELAND, 2003). De este modo muchos Planes Estratégicos se quedan sólo en un ejercicio voluntarista y nuca llegan a implantarse, por eso el análisis del proceso de Planificación Estratégica en Alcobendas es relevante ya que su Ayuntamiento es uno de los mejores exponentes de modernización administrativa con la introducción exitosa en el ámbito público local de instrumentos innovadores de gestión pública, entre los que se encuentra el diseño, implantación y evaluación de un Plan Estratégico.

Por lo demás, el estudio de caso ${ }^{2}$ tiene interés porque el proceso de planificación estratégica en Alcobendas se sustenta en el entendimiento de este mecanismo de gestión como

\footnotetext{
${ }^{2}$ Básicamente, la metodología que se utiliza en la investigación acerca de la Gestión Pública es una metodología compartida con otras Ciencias Sociales (PERRY y KRAEMER, 1990). Tanto en el ámbito de la gestión privada como en la gestión pública, la investigación se sustenta en diversas estrategias (BEHN, 1993). El diseño experimental como técnica de investigación, aunque de elegancia teórica, es difícil de implementar y más en el ámbito público, donde ejercer el control sobre las variables relevantes es un ejercicio imposible. El uso de cuestionarios no ayuda mucho a generar conocimiento relevante en cuanto que los gestores tienen más un conocimiento fenoménico que contextual de su trabajo (MINTZBERG, 2002). Por lo que hace a las técnicas de investigación cualitativa, la observación participante y las entrevistas requieren tener en cuenta el problema de la selección y la fiabilidad en cuanto a la información recogida, tal y como lo pusieron de manifiesto Miller y Mintzberg en sus trabajos acerca del "Executive Management" (MILLER y MINTZBERG, 1983). En este contexto, un estudio de caso, no sólo proporciona hechos relevantes sino que ayuda a identificar causas subyacentes capaces de explicar por qué una combinación de acciones que interactúan en circunstancias delimitadas conducen a determinados resultados de los que pueden deducirse principios aplicables a otras situaciones. Un ejemplo de ello vendría representado por las investigaciones de Kaufman (KAUFMAN, 1960). Por ello, el estudio de caso viene siendo reconocida como una de las mejores estrategias de investigación en el ámbito de la Gestión Pública para generar conocimiento de validez científica (AGRANOFF, 1986; McGREGOR, 1993; BEHN, 1993; LYNN, 1996). El razonamiento inductivo viene siendo utilizado a lo largo y ancho de diversos campos científicos. La investigación sustentada en un estudio de caso se sostiene en la premisa de que para generar teorías relevantes para la práctica efectiva de la gestión, es preciso identificar y sistematizar las observaciones que se dan en la práctica. Los elementos comunes que salen a la luz del estudio de casos, convenientemente sistematizados, conforman los cimientos para la elaboración de teorías válidas. Así, nos encontraríamos en el ámbito de lo que ARGYRIS, PUTNAM y McLAIN SMITH (1985) denominan como "action science", esto es, ciencia que se enriquece a través del estudio de las prácticas consideradas como más relevantes. De manera que este modo de proceder en la investigación tendría dos objetivos: por una parte, la generación de conocimiento científico fiable y válido y, por otra, proporcionar a los gestores una ayuda para una mejor comprensión de las situaciones en las que se ven involucrados. En la ciudad de Alcobendas se ha desarrollado en las últimas décadas un proceso de gobernanza propio, endógeno, que ha tenido como eje la Planificación
} 
canal de vehiculación centrípeta de las demandas políticas locales que se incardinan con los objetivos políticos del gobierno local. Asimismo, se establece un vínculo entre los objetivos políticamente determinados y su ejecución administrativa y que se hace posible en razón de las relaciones entre el liderazgo político, directivo y técnico.

\section{EL PROCESO DE PLANIFICACIÓN ESTRATÉGICA (PLAN CIUDAD) EN ALCOBENDAS: ESTRUCTURA Y FASES}

Desde los primeros años de la década de los 80 del pasado siglo, el equipo de gobierno adquiere un compromiso para, al tiempo que se consolida la democracia local, potenciar la provisión de servicios públicos locales para la mejora de las condiciones de vida de sus ciudadanos. Se adopta, por tanto, un modelo de gobernanza pública como un proceso continuo en el tiempo que implica un solapamiento de actuaciones de innovación en la gestión que se integran, engranan y superponen unas con otras y que podrían subdividirse en tres fases. Una primera fase (1984-1991), caracterizada por las reformas internas y por la puesta en marcha de la Dirección por Objetivos que sirve de soporte a los Planes Integrales de carácter sectorial y a partir de los cuales se implanta una nueva cultura organizativa. Una segunda fase (1991-1995), caracterizada por la elaboración de un Plan Estratégico de Ciudad a partir de la experiencia de los Planes sectoriales. Esta fase está caracterizada por la definición de un modelo de gestión que acentúa la dimensión omnicomprensiva de toda la organización municipal. Por sus características nos detendremos en el análisis de esta segunda fase en la que se pone en marcha el primer Plan Estratégico. Finalmente, una tercera fase (desde 1995 hasta la fecha), caracterizada por la preocupación constante por la calidad como mejora continuada. Esta tercera fase es el de la consolidación del modelo de gobernanza. Es de destacar que en 2007 se produjo un cambio en el color político del partido de gobierno, situación que no ha modificado en lo sustancial el proceso de modernización administrativa, centrada ahora en la impulsión de la Calidad a partir de los lineamientos diseñados en el último Plan Estratégico.

Estratégica. Se trata de un caso paradigmático que es interesante estudiar y analizar para dar respuesta a preguntas relevantes, entre otras a por qué y cómo se implanta un modelo de Planificación Estratégica que se enmarca en un proceso de gobernanza local y del que se deriva un proceso de modernización administrativa con éxito aparente. Para indagar en las cuestiones que nos interesan se ha estrucuturado de forma sistemática un amplio conjunto de fuentes directas e indirectas procediendo a trabajar sistemáticamente con varios instrumentos de recogida de información: a) Entrevistas semiestructuradas con 60 actores críticos (stakeholders) tanto de la organización municipal como del sector empresarial local y de las asociaciones vecinales, la mayoría de ellos han participado en la elaboración e implantación del Plan Estratégico, pero otros declinaron en su momento la participación; para evitar posibles sesgos se han realizado cruces de información; b) Observación participante activa: para abordar el estudio, el investigador ha formado parte del Foro Ciudad del Plan Estratégico como observador participante lo que ha permitido observar el caso de forma intensiva durante un largo período de tiempo; c) Análisis documental: Se han analizado documentos administrativos, actas de reuniones, documentación de los Plenos, publicaciones del Ayuntamiento y de consultores externos referidas al proceso, entre otros; este análisis documental ha contribuido a la preparación de entrevistas y a corroborrar la información obtenida de otras fuentes al objeto de verificar sus consistencia con las entrevistas y observaciones; d) Archivos municipales: se han estudiado los organigramas, presupuestos y servicios relacionados con el proceso de planificación; e) Grupos de discusión: se ha tenido acceso al análisis de los grupos de discusión realizados por el Ayuntamiento y en aquellos aspectos específicos relacionados con la Planificación Estratégica. 


\section{a) La Estructura del Plan Estratégico}

En Alcobendas, a la luz de la experiencia con los Planes Sectoriales, sobre todo del Plan Joven, puestos en marcha con anterioridad y que se implantan mediante un sistema de Dirección y Programación por Objetivos, la Planificación Estratégica constituye el siguiente eslabón y tiene por objeto movilizar a los principales actores de la política, la administración, la economía y la ciudadanía local para contribuir al desarrollo socioeconómico de la ciudad. Esta participación en el Plan Ciudad ha permitido una visión global de los problemas locales, asumiendo, de alguna manera, en el ámbito local la gestión de la globalización. El objetivo principal de la Planificación Estratégica en Alcobendas ha sido la adaptación institucional al entorno mediante la planificación y organización del espacio local a través de la integración de las iniciativas de la sociedad civil local en la acción de la organización administrativa.

Siendo un instrumento para la planificación a largo plazo, la institución municipal asume el proceso de elaboración y se define como un Plan sustentado en la participación: "Alcobendas es un proyecto abierto, común, participado que debe ser desarrollado a través de un esfuerzo coordinado de todas las administraciones, instituciones, sector privado y sociedad civil"'3. Con todo, se introduce una triple innovación por cuanto las características del Plan Estratégico de Alcobendas son: 1) Liderazgo continuado, 2) Capacidad de movilizar actores económicos y sociales, 3) Las ideas estratégicas se combinan con actuaciones viables a corto plazo con resultados concretos, lo que dota al Plan de credibilidad y capacidad para transformar la ciudad.

En un proceso de Planificación Estratégica resulta capital la determinación de quién participa y de cómo se establecen los mecanismos de comunicación y de adopción de decisiones en el proceso (FUNG, 2006). A partir del análisis empírico de los documentos de Planificación Estratégica en Alcobendas se puede concluir que el modelo se sustenta sobre todo en la movilización de los siguientes grupos de actores estratégicos: a) vecinos interesados en los asuntos públicos y que están dispuestos a actuar de representantes de un conjunto más amplio de vecinos (asociaciones) actores; b) representantes de los intereses organizados en la ciudad (empresarios y ONG's); c) cargos electos y partidos políticos; d) gestores y representantes de los empleados públicos; e) expertos técnicos. Esto es, la Planificación Estratégica se concibe como un proceso de participación concertada de un amplio número de agentes del entramado político, económico y social de la ciudad que se ocupan de los temas que afectan al futuro de la ciudad más allá de una legislatura. De esta manera, el Plan Estratégico de Alcobendas (Plan Ciudad) es el resultado del trabajo realizado durante un período de dos años por 124 representantes institucionales, 122 asociaciones, fundaciones y agentes sociales y 70 empresarios. Todos estos actores son liderados por el Ayuntamiento de Alcobendas que constituye un Comité Ejecutivo en el que están representados la pluralidad de intereses de la ciudad (DOCUMENTO ALCOBENDAS PLAN CIUDAD).

Por lo demás, en la elaboración del Plan se observan dos niveles de participación de la sociedad civil local: 1) en el desarrollo de una "visión" de la ciudad a largo plazo, de tal for-

\footnotetext{
${ }^{3}$ Fuente: "Plan Ciudad, modernización y calidad en la gestión" en Alcobendas Plan Ciudad, Calidad y Modernización en la Gestión Pública, Ayuntamiento de Alcobendas.
} 
ma que se plantea a un plazo de diez años el futuro de la ciudad; 2) en el diseño de proyectos concretos, que se llevan a cabo y que no crean falsas expectativas para lo que, en el marco de las estrategias del Plan Ciudad, se presentan 71 proyectos concretos para su valoración técnica, ciudadana y del Comité Ejecutivo y posteriormente presentados a la Asamblea General del Plan que aprueba 69 de aquéllos con el compromiso de desarrollarlos de acuerdo con el nivel de prioridad de cada uno de ellos. Asimismo, de la lectura del Plan Ciudad se deduce que aborda asuntos territoriales, económicos y socioculturales. Su articulación gira en torno a dos ejes: el desarrollo social y económico sostenible, definiéndose un modelo de ciudad y la consecución de un objetivo: "Una ciudad ecológica, con un desarrollo sostenible para crear empleo y equilibrado con el medio ambiente, que mantenga y mejore el bienestar y calidad de vida actual, con la participación de todos y con un fuerte protagonismo al Norte de Madrid".

Es, por tanto, un proceso participativo en el que, teniendo también en cuenta la consulta a una muestra aleatoria estratificada de 4.017 vecinos, se definen diferentes líneas estratégicas. En el proceso de elaboración e implantación del Plan Estratégico se pueden identificar aspectos de información, de consulta, y de concertación. La participación también tiene lugar en el nivel de elaboración de los proyectos concretos y en aspectos de su implantación a través del tejido asociativo.

Por otra parte, la decisión de hacer Planificación Estratégica se contempla en el Plan de Acción Municipal 1992-1995 y, con objeto de dotar de un sustento normativo al Plan Estratégico, se le hace coincidir con el Plan General de Ordenación Urbana. Sus estrategias están ligadas al Plan de Acción Municipal que, a su vez, está incardinado con el Presupuesto. Al estar ligado con el proceso presupuestario las acciones contempladas en el Plan son posibles y congruentes.

\section{b) Las fases del Plan Estratégico}

A partir de la metodología que permite hacer compatibles la participación y la eficacia, se establecen distintas fases de actuación. En una fase preliminar desde el equipo de gobierno se recopila información y análisis de experiencias similares llevadas a cabo en otras ciudades. En la primera fase, que se inicia en Julio de 1993, se elabora la metodología que es aprobada por el Pleno Municipal ${ }^{5}$, al tiempo que se crea una Oficina Técnica del Plan encargada de realizar un diagnóstico acerca de la realidad social, económica y territorial de la ciudad en el que participan especialistas, realizando informes que den cuenta de la situa-

\footnotetext{
${ }^{4}$ En ese Plan de Acción Municipal se incluye la realización de un Plan Estratégico con el objetivo de "Diseñar el futuro de la ciudad como elemento integrador y orientador de los diferentes agentes económicos, sociales e institucionales".

${ }^{5}$ Con el apoyo por unanimidad de todos los miembros del Pleno, el 13 de Julio de 1993 se aprueba el Plan Estratégico que tiene como finalidades: a) la optimización de la posición de la ciudad en su territorio para la mejora de la eficacia productiva, la cohesión social y la calidad de vida de los ciudadanos, b) la realización de un diseño de futuro de ciudad en el horizonte del año 2000, integrando los grandes proyectos urbanísticos, económicos, socioculturales en marcha, articulando el presente y futuro de la ciudad en un proyecto creíble y posible, c) conseguir que el conjunto de la organización municipal e instituciones trabaje de forma integrada hacia objetivos globales. (Fuente: Balance Plan Ciudad 1995-2002, Dirección de Planificación y Coordinación, Documento de Trabajo para la Comisión Ciudadana, Diciembre de 2002, mimeografiado).
} 
ción actual de la ciudad. Desde esta Oficina Técnica se lanza la imagen del Plan Ciudad y se le vincula a otros proyectos en marcha. Se crea una marca para identificar el proceso de reflexión (Alcobendas Plan Ciudad) y desde la Oficina Técnica se producen acciones comunicativas mediante comparecencias públicas de los responsables políticos, anuncios en prensa, vallas publicitarias, cuñas radiofónicas y publicaciones municipales, entre otras.

Durante la segunda fase, que tiene lugar a principios de 1994, y para el diseño, elaboración e implantación de forma participada del Plan Estratégico, se crea una estructura organizativa como soporte al proceso de elaboración, implantación, seguimiento y evaluación del Plan. A partir de ahí, se definen, mediante un proceso de reflexión en el que se involucra a los actores, las estrategias que conforman la "visión" de futuro de la ciudad. A estos efectos, se constituye el Comité Ejecutivo para la dirección del Plan y la Asamblea General así como las Mesas Temáticas, se realizan consultas a los vecinos de la $\operatorname{ciudad}^{6}$, y se define el perfil de los distintos proyectos, tanto por parte del Comité Ejecutivo como de la Oficina Técnica del Plan.

Finalmente, hay una fase de implantación, seguimiento y evaluación del conjunto de acciones que abarca el Plan Estratégico. Para ello se establecen una serie de indicadores que permiten recabar información para el control del grado de realización de los proyectos asociados a cada una de las líneas estratégicas. A partir de esta información se toman decisiones apropiadas para garantizar su cumplimiento.

\section{c) Las líneas estratégicas del Plan}

A partir de una evaluación de las carencias y potencialidades de la ciudad de Alcobendas se desarrolla una visión estratégica de la ciudad y se definen y priorizan un conjunto de líneas estratégicas para alcanzar esa visión. En el establecimiento de objetivos estratégicos se da un papel relevante a los diferentes actores que intervienen en el Plan. A partir de aquí, estos objetivos son priorizados por la dirección política ${ }^{7}$ y se vinculan a acciones operativas. Se tienen en cuenta las necesidades de los ciudadanos identificadas a través de las citadas 4.017 encuestas que se realizan a los vecinos de Alcobendas.

De este modo, bajo un objetivo general o misión que atiende a optimizar la posición de Alcobendas para mejorar su eficacia productiva, la cohesión social y la calidad de vida de los ciudadanos, en el Plan Ciudad de Alcobendas se pueden identificar cinco actuaciones estratégicas en las que se enmarcan 69 propuestas de proyectos de actuación concretos y que, a su vez, se organizan en tres niveles atendiendo a la prioridad en su realización. A este respecto, el Alcalde se compromete a la realización de los proyectos emanados del Plan Ciudad.

\footnotetext{
${ }^{6}$ Se realizó una encuesta a más de 4000 ciudadanos para conocer su opinión sobre los objetivos y propuestas del Plan Ciudad. Ésta puso de manifiesto que el $76 \%$ de los ciudadanos, a los que se sometió a la encuesta, pensaba que con la puesta en marcha del Plan y sus proyectos la ciudad mejoraría.

${ }^{7}$ Estos objetivos son: a) la optimización de la posición de Alcobendas en el territorio desde una perspectiva regional en el marco de los sistemas de ciudades español y europeo, b) el diseño de un proyecto de ciudad para los próximos diez años, c) la integración de las acciones ya en marcha referidas a proyectos urbanísticos, económicos y socioculturales, d) la articulación del conjunto de organismos e instituciones con interés en la ciudad (Documento elaborado por la Oficina Técnica del Plan Ciudad).
} 
En estas condiciones, se establecen las siguientes líneas estratégicas encaminadas a la consecución del objetivo general: 1) "Bienestar y calidad de vida, desarrollo sostenible" en la que se define el modelo de desarrollo de ciudad y que se incorpora al Plan de Ordenación Urbana. Alcobendas se adhiere a la Carta de Aalborg para implantar la Agenda Local 21. En esta línea estratégica se engloba la promoción del desarrollo equilibrado de la ciudad, de sus ciudadanos y su entorno medioambiental. Las actuaciones en materia medioambiental han supuesto el otorgamiento del premio "Bandera Verde-Ciudad Sostenible" que otorga la Federación de Usuarios-Consumidores y la mención "good" en el Concurso de Naciones Unidas sobre Buenas Prácticas para la Mejora del Entorno Urbano dentro del apartado de Gestión sostenible de los Recursos Naturales. 2) "Alcobendas, el Norte de Madrid" para situar a la ciudad en el sistema metropolitano madrileño aprovechando su situación al norte de Madrid. 3) "Dinamismo económico y empleo" bajo la que se busca la atracción de inversiones de empresas del sector de la sociedad de la información y del conocimiento para potenciar el dinamismo económico para la creación de empleo, reequilibrando el binomio residencia-empleo y conseguir altos niveles de integración social. 4) "Personas preparadas" para la mejora permanente de la formación y cualificación de las personas y 5) “Alcobendas, entre todos mejor" para abrir la ciudad y la institución municipal a la cooperación y a la participación al tiempo que se busca la calidad en la prestación de los servicios públicos (DOCUMENTO BALANCE PLAN CIUDAD).

\section{LA ESTRUCTURA ORGANIZATIVA DEL PLAN}

La elaboración del Plan exige mantener la movilización de los actores críticos y que estos cuenten con mecanismos que les faciliten su relación e interacción para suministrar diferentes "inputs" y mantener vivo su compromiso para la implantación del plan. Pero más acá de la creación de estructuras está la necesidad de que exista un liderazgo claro capaz de aglutinar intereses para el diseño de las estrategias. En Alcobendas este liderazgo es ejercido por el Primer Teniente de Alcalde quién compagina el liderazgo político con la profesionalización ${ }^{8}$. A partir de aquí, el Plan Estratégico de Alcobendas se estructura en torno a la siguiente organización:

La Asamblea General del Plan Ciudad, que se constituye como máximo órgano de participación institucional y ciudadana. Aglutina a los actores relevantes para la ciudad e incluye a agentes de los ámbitos político, económico y del tejido asociativo. A la Asamblea se invita a participar a 345 miembros que reúnen estas características y aceptan su inclusión en el proceso. Finalmente a la primera Asamblea acuden 208 personas que representan a distintas instituciones, asociaciones, fundaciones, empresas

\footnotetext{
${ }^{8}$ En el papel del Primer Teniente de Alcalde hay un cierto isomorfismo con otras figuras existentes en los países de tradición anglosajona y del Norte de Europa. Así, en el ámbito de las ciudades de tamaño medio norteamericanas, existe la figura del el gerente municipal, City Manager, quien es un líder administrativo pero en interacción continua con los políticos locales, teniendo un papel relevante en el proceso de gobierno local, de tal manera que ejerce una influencia importante en la elaboración y en la implantación de las políticas públicas locales al tiempo que se ocupa del establecimiento de diseños organizativos como instrumentos facilitadores de la consecución de los objetivos de las políticas públicas municipales.
} 
y comercios minoristas. Esta Asamblea tiene como función la aprobación de las directrices finales del Plan; es el referente y responsable del impulso del mismo. Es un foro de debate abierto a la participación de nuevos actores en función de la evolución del proceso. Al plenario de la Asamblea se presentan los avances del Plan a cuyo tenor, si es necesario, se replantean las líneas estratégicas.

El Comité Ejecutivo del Plan Ciudad, es un órgano plural que consta de 16 miembros y está presidido por el Alcalde ${ }^{9}$, actuando de vicepresidente ejecutivo el Primer Teniente de Alcalde, quien es responsable del proceso de elaboración del Plan y de su financiación o vinculación a la planificación presupuestaria plurianual, es decir, del control del proceso y de la elaboración de los temas estratégicos. Desde este Comité se dirige a las Mesas Temáticas y se aprueban los documentos generados por éstas. Además de las personas con responsabilidades políticas, en este órgano están representados y se concentran miembros pertenecientes al mundo de la empresa, los agentes sociales y los vecinos a través de sus asociaciones. Así, además del Ayuntamiento de Alcobendas, forman parte la Asociación de Empresarios, la Cámara de Comercio e Industria de Madrid, la Comunidad de Madrid, Sindicatos y Consejos Sectoriales Ciudadanos.

La Oficina Técnica del Plan Ciudad, en la que se integra la totalidad del Departamento de Planificación y Coordinación del Ayuntamiento dependiente del Primer Teniente de Alcalde. Su equipo gestor está encargado del impulso y de la coordinación de la elaboración e implementación del Plan Ciudad; esta función de coordinación incluye la supervisión de los estudios para el diagnóstico estratégico y la elaboración de los informes que habrían de servir de base a las deliberaciones de las Mesas Temáticas. Su papel es primordial ya que actúa como centro vital del Plan. Está encargada de la dinamización del proceso y se responsabiliza de que las estrategias se desarrollen mediante la implantación de los proyectos asociadas a las mismas. Actúa como centro de referencia para todos los actores participantes en el seguimiento de las decisiones que se tomen en la Asamblea y se encarga del almacenamiento y difusión de la información del Plan. Organiza y participa en las Mesas Temáticas, identificando los actores críticos que deben participar en las mismas y los proyectos relevantes a los que da coherencia. Proporciona información a los cargos electos que les sirve de soporte en los procesos de negociación con los diversos actores involucrados en el Plan. Se ocupa también de las labores de comunicación del Plan tanto dentro de la ciudad como fuera a través de la participación en foros y encuentros y recoge información de experiencias similares en otras ciudades. Elabora propuestas para la priorización de los proyectos y prepara indicadores de cumplimiento del Plan y realiza un seguimiento del estado de evolución

\footnotetext{
${ }^{9}$ Los cargos que ostentan los 16 miembros de este Comité Ejecutivo son: Presidente, Alcalde de Alcobendas, Vicepresidente, Primer Teniente de Alcalde de Alcobendas, Secretario, Director de la Oficina del Plan Estratégico, Vocales: Director del Instituto Madrileño de Desarrollo, Consejo Sectorial de Deportes, Consejo Sectorial del Medio Ambiente, Consejo Sectorial Juventud y Mujer, Director de Planificación Urbanística de la CAM, Consejo Sectorial de Cultura, Teniente Alcalde Ayuntamiento de Alcobendas, Secretaría Comarcal de CC.OO. Teniente de Alcalde Ayuntamiento de Alcobendas, Instituto Madrileño de Formación, CAM, Secretario Comarcal de UGT, Presidente Asociación de Empresarios de Alcobendas, Delegada de la Cámara de Comercio e Industria de Madrid. La composición pone de manifiesto la mayoría de cargos políticos de la institución promotora. (Fuente: Alcobendas Plan Ciudad).
} 
de las distintas líneas estratégicas y proyectos. De modo que este órgano condiciona las interacciones entre la Asamblea, el Comité y la propia Oficina Técnica.

Complementariamente a los órganos de representación y ejecutivos, se crean cuatro órganos de consulta y participación: Las Mesas Temáticas, el Grupo de Estudios, la Comisión de Grupos Políticos y la Comisión de Directivos Institucionales.

a) Las Mesas Temáticas, que implican a miembros de la Asamblea y se corresponden con la estructura de poder de la ciudad y tienen la función de elaborar y proponer proyectos o modificar o ratificar los que se les presentan.

Con esta composición, son grupos de trabajo de ámbito sectorial para la participación y el debate. Su característica es la complementariedad, es decir, que aglutinan a actores con intereses distintos y actúan de un modo colaborativo y corresponsable. Se constituyen doce de estas Mesas ${ }^{10}$ en las que trabajan 378 personas pertenecientes a los ámbitos empresariales y sindicales, comerciantes, representantes de entidades sociales y culturales, universidades, representantes de otros Ayuntamientos y de la Comunidad de Madrid, junto con los miembros del Comité Ejecutivo. Cada Mesa está presidida por un miembro del Comité Ejecutivo del Plan y asiste a cada una de ellas un experto convocado como tal. A los participantes la institución municipal les facilita todo tipo de apoyo técnico indispensable para el desarrollo de las deliberaciones. A las reuniones de todas las Mesas asiste como ponente el Director de la Oficina Técnica del Plan Ciudad. En todas las Mesas se utiliza la misma metodología de análisis, consistente en debatir acerca de la situación actual del tema a tratar, las tendencias e impactos previsibles y la reflexión acerca de los puntos fuertes y débiles de los aspectos a considerar.

De este modo, las Mesas Temáticas son grupos de trabajo especializado e instrumentos plebiscitarios a la vez que sirven para institucionalizar estructuras de participación. Se ponen en marcha doce mesas que se constituyen en el instrumento operativo para la elaboración del Plan, identificando proyectos específicos vinculados a las diferentes líneas estratégicas. La implantación de estos proyectos se prioriza en función de la importancia, de sus efectos multiplicadores o por tratarse de proyectos ya en fase de ejecución. Al mismo tiempo, se identifican indicadores de implantación de cada uno de los proyectos.

Las Mesas se reúnen en fechas definidas durante cinco meses y en ellas los participantes recibieron apoyo técnico mediante la aportación de estudios, informes y documentos de trabajo elaborados por la institución municipal. Cada una de las doce

\footnotetext{
${ }^{10}$ Estas doce Mesas Temáticas se constituyen para debatir acerca de las siguientes políticas sectoriales: 1. Desarrollo urbano y calidad medioambiental, en la que se inscriben 45 personas, con 33 personas inscritas, 2. Calidad de Vida y nuevas demandas, en la que participan 41 personas, 3. Seguridad Ciudadana, que reúne a 31 personas, 4. Integración Social, con 39 personas inscritas 5. Accesibilidad, con una media de 42 personas, 6. Promoción de la Ciudad y sus dotaciones, con 54 personas, 7. Atracción de la inversión externa, en la que se inscriben 35 personas, 8. Impulso de la economía local, que convoca a 26 personas, 9. Sistema educativo y vinculación de la ciudad a las universidades, en la que participan 59 personas, 10. Formación y orientación laboral, con 51 personas, 11. Modernización y participación institucional, en la que se inscriben 37 personas, 12. Asociacionismo y voluntariado, en la que se inscriben 54 personas. En las Mesas intervienen los agentes sociales y culturales más activos de la ciudad junto a los representantes empresariales y sindicales. (Fuente: Alcobendas Plan Ciudad: Calidad y Modernización en la Administración Pública. Ayuntamiento de Alcobendas-Ediciones Gestión 2000.
} 
mesas estaba presidido por un miembro del Comité Ejecutivo del Plan (en siete de ellas el presidente era un cargo político) y en todas estaba representada la Administración local por medio del Director de la Oficina del Plan Ciudad de Alcobendas, que era al tiempo Director del Departamento de Planificación y Coordinación. Los participantes trabajaban sobre un esquema de Tendencias-Puntos Fuertes y Puntos Débiles siguiendo una metodología preparada por la institución municipal presentándose pocos problemas y con un elevado grado de consenso y de compromiso. En este sentido, las Mesas pueden considerarse como un instrumento en el que se resuelven los conflictos mediante la cooperación en el marco de una estrategia de creación de redes entre la Administración y la ciudadanía. La existencia de una mayoría absoluta permite que se generen estos ámbitos de conflicto.

A través de las Mesas Temáticas se institucionalizan estructuras de participación que se reúnen en días definidos. Estas Mesas son moderadas de tal forma que todos los participantes presenten propuestas y las desarrollen. Así, bajo la moderación y organización de la Dirección de coordinación se debate acerca de los problemas locales, y está abierta a la participación de cualquier vecino. Las Mesas se constituyen en un instrumento para la gestión política que conduce a resultados evaluables por los ciudadanos y los usuarios específicos. En las mesas se inscribieron un total de 539 personas, de las que activamente participaron 378, incluyendo al Alcalde, cargos políticos, y representantes de intereses empresariales, sindicales y vecinales; los participantes han mantenido docenas de reuniones durante un elevado número de horas de trabajo. El número de participantes hace indicar que no ha existido un dominio de intereses particulares, dejando fuera influencias clientelares. Se deja claro desde el principio que estas mesas no sustituyen el proceso político de toma de decisiones. Es cierto que los grupos en las Mesas representan a intereses particulares y en este sentido debe tenerse en cuenta el total del entorno dentro del cual existen estos intereses especiales. En eso es precisamente en lo que consiste la dirección a través de la coordinación que ejerce la Oficina Técnica del Plan. Las propuestas de las Mesas Temáticas se coordinan mediante un sistema de prioridades.

b) El Grupo de Estudios está formado por los directores de las investigaciones de base que proporcionan a los participantes información y orientaciones metodológicas. Sus miembros pertenecen al equipo directivo de la institución municipal y, puntualmente, cuentan con la ayuda y asesoramiento de especialistas externos a la organización municipal.

c) La Comisión de Grupos Políticos está compuesta por los portavoces de los grupos políticos con representación en el Pleno Municipal y actúa como órgano de consulta para el Comité Ejecutivo.

d) La Comisión de Directivos Institucionales está formada por directivos del Ayuntamiento y tiene como función la coordinación de las líneas estratégicas del Plan así como la implantación de las mismas. Se constituye en un instrumento para la implicación del nivel directivo en la Planificación Estratégica.

Todos estos órganos intervienen en la redacción y priorización de los proyectos, lo que permite que en marzo de 1995 se apruebe el Plan en Asamblea General, se 
presente a los ciudadanos y se proceda a su ejecución por parte del equipo de gobierno y de la Oficina Técnica del Plan ${ }^{11}$, de manera que los proyectos aprobados se van integrando según orden de prioridad en los Planes de Acción Municipal de las diferentes legislaturas.

\section{LA IMPLANTACIÓN Y EVALUACIÓN DEL PLAN ESTRATÉGICO}

La implantación es un aspecto clave pues, como se ha dicho al principio, muchos planes estratégicos locales no llegan más allá de la fase de diseño. Por otra parte, la evaluación es necesaria no sólo porque el proceso de planificación exige el esfuerzo de múltiples actores y sea preciso conocer sus resultados, sino también porque la transparencia y la dación de cuentas deben ser consustanciales a la acción pública. Con ello en mente, en Alcobendas los proyectos aprobados en el Plan Estratégico se ponen en marcha para ser realizados en un plazo de diez años mediante su inclusión, como se acaba de indicar, en los sucesivos Planes Municipales.

En Abril de 1997 se constituye el Foro Consultivo del Plan Ciudad con el objetivo de formar un espacio de participación, debate e impulsión del Plan Ciudad. Este Foro se constituye con objeto de realizar el seguimiento de la ejecución del Plan Estratégico. En este Foro se integran todos los que en su momento constituyeron la Asamblea General del Plan así como nuevas incorporaciones.

De esta manera, el Foro Ciudad, se concibe como un instrumento para hacer el seguimiento y evaluación de todos los proyectos del Plan Estratégico, a la vez que un instrumento de participación, articulado con el resto de instrumentos en marcha en Alcobendas, a partir del cual se pretende dar cuentas a los ciudadanos acerca de las realizaciones del Plan Ciudad para que emitan una valoración. Al tiempo que se hace esa valoración, se requiere a los ciudadanos para que, a partir de las realizaciones, se diseñe el futuro de la ciudad, manteniendo así vivo y actualizado el Plan Estratégico. El Primer Foro Ciudad se reúne por primera vez en marzo de 1999, a los dos años del diseño de la finalización del Plan Estratégico y continúa reuniéndose con periodicidad bianual. Se compone de ciudadanos, asociaciones, empresarios, comerciantes y fundaciones. Pretende involucrar a los ciudada-

\footnotetext{
${ }^{11}$ La tercera etapa del Plan Ciudad se pone en marcha con la presentación de proyectos por parte de los distintos actores implicados en el Plan Ciudad. El total de proyectos recibidos fue de 71, de los cuales el 31\% los presentó el Ayuntamiento de Alcobendas, el 30\% las asociaciones vecinales, el 18\% las Mesas Temáticas, el 16\% otras administraciones públicas y el 11\% empresas y asociaciones de empresarios. Cada uno de estos proyectos se sometió a la valoración de los miembros de la Asamblea en función de unos criterios mediante los cuales se valoraban sus impactos en el desarrollo económico, ambiental, cohesión social y plausibilidad del proyecto. Una vez realizada la valoración por la Asamblea del Plan, esta trasladó sus conclusiones a la Oficina Técnica del Plan para la elaboración de un informe técnico referido a cada uno de los proyectos. Estos informes técnicos fueron remitidos, finalmente, al Comité Ejecutivo para una ulterior valoración teniendo en cuenta el grado de complementariedad con otros proyectos, el consenso de los implicados en su realización y financiación, la existencia de desarrollos técnicos avanzados y la urgencia de cada uno de los proyectos. El Comité Ejecutivo descartó por unanimidad dos proyectos y el resto los agrupó en tres grupos o niveles de prioridad que fueron presentados finalmente a la Asamblea para su aprobación definitiva. El proceso de aprobación de proyectos pone de manifiesto el control, también en esta fase, de las instancias políticas y técnicas del Ayuntamiento por medio de sus órganos de representación en el Plan. (Fuentes: Alcobendas Plan Ciudad y Balance del Plan Ciudad 1995-2007).
} 
nos que han participado en el Plan Estratégico de Ciudad y a aquellos que se incorporan nuevos, tanto los encuadrados en las asociaciones como a los que no pertenecen a ellas.

Los objetivos del Foro del Plan Ciudad son ${ }^{12}$ :

1. La reflexión y debate sobre el futuro de la ciudad.

2. La constitución de un espacio de encuentro entre la institución de gobierno municipal, los ciudadanos y los agentes empresariales y sindicales.

3. Realizar el seguimiento de los Proyectos del Plan Estratégico.

4. Crear y lanzar iniciativas relacionadas con los proyectos estratégicos de la ciudad utilizando para ello los denominados "Grupos de Impulsión” de proyectos. En este sentido se propone la creación de 3 grupos de trabajo, debate y seguimiento relacionados con los proyectos y prioridades estratégicas de la ciudad. El objetivo de estos grupos es la generación de consensos entre las instituciones político-administrativas, la sociedad civil y los empresarios encaminados a la realización de los proyectos así como la identificación de nuevas oportunidades para el desarrollo de la ciudad. De este modo se configuran tres grupos:

a) El grupo de impulsión "Alcobendas Eco-Ciudad" cuyo objetivo es impulsar el modelo de desarrollo urbano, cualitativo y sostenible delineado en el Plan Ciudad de Alcobendas, contribuyendo a la implantación de los distintos proyectos que lo sustentan así como su posible desarrollo. Los Proyectos implicados en este grupo son un modelo de desarrollo urbano del Plan General de Ordenación Urbana, Tren de Cercanías, Proyectos urbanísticos, reutilización de aguas residuales, recuperación de espacios naturales en la ciudad y educación medio ambiental.

b) El Grupo de Impulsión "Alcobendas Casco Antiguo", cuyo objetivo es el impulso de la revitalización de los barrios que conforman el Casco Histórico de la ciudad de acuerdo con un modelo de desarrollo urbano cualitativo y sostenible que se define para el conjunto de la ciudad, teniendo como proyectos la rehabilitación del Casco Viejo, la creación de un bulevar en la antigua carretera nacional y en general, mejorar la accesibilidad.

c) El grupo de Impulsión "Alcobendas Calidad" con el objetivo de distinguir a Alcobendas como "Ciudad de Calidad" y a la institución municipal como entidad dedicada al servicio de los ciudadanos, generando al mismo tiempo una imagen de ciudad para la atracción de inversiones, la actividad económica y como ciudad de referencia distinguida por su oferta de actividades culturales y deportivas. Los proyectos son la Calidad Total en el Ayuntamiento, la promoción de la ciudad y su inclusión en redes de ciudades, el fomento de la participación ciudadana y la creación de espacios culturales.

Con estas premisas se invita a los dirigentes empresariales y sindicales así como a representantes del movimiento ciudadano y demás instituciones con vinculación en la ciudad de Alcobendas a formar parte del Foro.

En marzo de 1999 se realiza una primera evaluación de resultados en el marco del Foro Consultivo del Plan Ciudad; en ese momento estaban implantados el 64\% de los proyectos

\footnotetext{
${ }^{12}$ Fuente: Constitución del Foro Consultivo del Plan Ciudad de Alcobendas. Ayuntamiento de Alcobendas. Abril 1997.Documento interno.
} 
aprobados. En el año 2001 se pone en marcha el Segundo Foro Ciudad. Para la evaluación de lo realizado por parte de los ciudadanos y la presentación de nuevas propuestas el $2^{\circ}$ Foro Ciudad se organiza un Pleno, una Comisión Ciudadana y tres Comisiones de Trabajo: Desarrollo Económico y Empleo, Cohesión e integración social y Calidad de Vida y Medio Ambiente. A las sesiones del Pleno del Foro Ciudad pueden asistir todos los ciudadanos. Se celebran sesiones en Enero y Marzo de 2003. La convocatoria de estas sesiones se hace de tal manera que tenga la mayor difusión posible y sea conocida por el mayor número de ciudadanos. La Comisión Ciudadana se conforma a partir de los miembros del Comité Ejecutivo del Plan Ciudad más otras instituciones vinculadas a las líneas estratégicas de la ciudad o que ejercen un liderazgo social y económico en la ciudad. Esta Comisión tiene como cometido la revisión y evaluación de los proyectos del Plan Ciudad. Las Comisiones de Trabajo pretenden recabar las aportaciones de todos los ciudadanos que intervienen en el proceso. Al final del proceso se edita un Documento de Aportaciones en el que se recogen las propuestas de los participantes acerca de las actuaciones que debieran diseñar el futuro de la ciudad. Con objeto de realizar un balance de la situación estratégica de Alcobendas y entrega del conjunto de aportaciones al Alcalde (Metodología y Reglamento del II Foro Ciudad de Alcobendas, Diciembre de 2002).

En junio de 2003 tiene lugar la segunda evaluación del Plan a través del Foro Ciudad, en ella intervienen las personas y entidades involucradas en el Plan Estratégico así como nuevos ciudadanos a título individual o encuadrados en asociaciones y actores económicos. Se elabora un documento que se dirige al equipo de gobierno y a la ciudadanía en general, habiéndose realizado hasta esa fecha el $87 \%$ del conjunto de los proyectos previstos ${ }^{13}$.

\section{LOS CRITERIOS DE ÉXITO DEL PLAN ESTRATÉGICO DE ALCOBENDAS}

El Plan Estratégico de Alcobendas cuenta con una doble legitimación, ex ante: se invita a participar a los representantes de los intereses; y ex post: la realización efectiva de los decidido en el espacio de deliberación con un alto grado de cumplimiento en Alcobendas. Se decide llevar a cabo 57 proyectos, de los cuales, como se acaba de indicar, en 1999 se llega a un grado de cumplimiento del 64\% y a finales del año 2002 están implantados el 87\% de los proyectos ${ }^{14}$. Los ciudadanos individuales están informados acerca de la existencia del Plan mediante comunicaciones individuales y a través de las revistas de información municipales, además de la información a través de los Consejos Sectoriales y los Consejos de Barrio.

\section{a) Un Plan percibido como útil para la ciudad}

Además de instrumento para aglutinar las iniciativas de los actores locales y para la determinación de prioridades, el Plan Estratégico tiene en cuenta el conjunto de problemas

\footnotetext{
${ }^{13}$ Fuente: Balance Plan Ciudad 1995-2002.

${ }^{14}$ Fuente: Balance Plan Ciudad 1995-2002. Dirección de Planificación y Coordinación. Documento de Trabajo para la Comisión Ciudadana. II Foro Ciudad, Diciembre 2002. Ayuntamiento de Alcobendas, mimeografiado.
} 
locales, incluyendo los aspectos más visibles y que afectan a la vida cotidiana de los ciudadanos. Contiene, por ende, una descripción cualitativa de la estrategia y hay un compromiso de vinculación al Plan de Acción Municipal.

\section{b) Un Plan realista}

Que se adecua a las características de la ciudad de Alcobendas y, conforme a ellas, se definen unos objetivos políticos claros y con el compromiso e implicación del Alcalde. c) Una administración profesional.

La administración dispone de capacidad para ejercer el liderazgo que se exige en las distintas fases del Plan; así, la Dirección de Planificación y Coordinación se ocupa del seguimiento y se responsabiliza de los trabajos y puesta en práctica y una gestión activa y transparente de la información acerca del proyecto y realmente abierta a la sociedad civil local.

\section{c) Interacción y comunicación}

El Plan se concibe como un proceso continuado en el tiempo y el que se implica a los actores con peso específico en la ciudad a la búsqueda de consensos. En la primera fase del proceso ciertos temas no se articulan. Se presenta un documento, elaborado por la Oficina Técnica del Plan, quien delinea las finalidades del mismo: optimización de la posición de la ciudad en el territorio del áreas metropolitana para mejorar la cohesión social, la eficacia productiva y la calidad de vida de sus ciudadanos; diseñar un proyecto de futuro de ciudad integrando los grandes proyectos urbanísticos económicos y socioculturales y lograr que el conjunto de la organización administrativa trabaje de manera integrada para la consecución de los objetivos globales ${ }^{15}$.

En lo referente a la inclusión y exclusión de temas, se desarrolla el Comité de Planificación y se decide quiénes participan. La responsabilidad de elegir las áreas de actuación y los participantes la tiene el Primer Teniente de Alcalde. Los participantes fueron seleccionados en función de la estructura de poder en Alcobendas. Todos fueron invitados a participar y, prácticamente, todos aceptaron. A los participantes se les pone a trabajar en temas específicos aunque las decisiones clave acerca de la planificación estratégica en sus distintas etapas se toman por parte del equipo de gobierno. En la primera etapa el Alcalde define el desarrollo de la estrategia cuyo primer aspecto prioritario es la revitalización del centro histórico. En la segunda etapa el Alcalde y el Primer Teniente de Alcalde proponen los temas generales a incluir en el proceso de planificación estratégica. Durante la siguiente etapa el Primer Teniente de Alcalde delimitan la agenda que es negociada por los miembros del Comité Ejecutivo con el resto de actores participantes.

De esta manera, sobre los temas estratégicos decide, en última instancia, el Comité Ejecutivo junto con la Oficina Técnica en función de unos criterios y en lo referente al control de la información, en la primera reunión del Plan, se transmite una gran cantidad de la mis-

\footnotetext{
15 Plan Ciudad de Alcobendas. Propuesta Metodológica Plan Estratégico de Ciudad. Documento mimeografiado, sin fecha. Oficina Plan Ciudad de Alcobendas.
} 
ma, de modo que a cada Mesa Temática se le proporciona un elenco de datos exhaustivos, aunque accesibles, de interés para los participantes.

\section{d) Alto nivel de implantación}

Que dota de credibilidad y continuidad al Plan y que genera un clima de confianza que a su vez moviliza a nuevos actores.

\section{e) Participación}

Aunque evaluar el impacto real de la movilización de la sociedad civil local no es fácil, lo cierto es que la reflexión estratégica sale del círculo de técnicos y se hace permanente e involucra a cada vez un mayor número de actores y ciudadanos que están satisfechos con el proceso ${ }^{16}$. Por ello, puede afirmarse que el proceso de participación ha sido prioritario a la definición de estrategias y proyectos, pensando en que su implantación sería dependiente de la calidad de la participación en el proceso, que ha servido de instrumento para integrar las relaciones fragmentadas y su puesta al servicio del logro de un modelo de ciudad.

\section{f) Cooperación}

El Plan parte del presupuesto de la importancia de identificar las relaciones de interdependencia entre los actores públicos y privados. Se trata de aglutinar el conocimiento local capaz de general sinergias para resolver los problemas locales, por lo que el proceso de participación se ha priorizado sobre la definición de estrategias y proyectos, no sin tener en cuenta que la implantación de éstos es dependiente del grado y la calidad de la participación en el proceso. La cuestión de quien toma las decisiones involucra a tres grupos: las elites políticas, los miembros del Comité o los ciudadanos. La formación de una Asamblea y un Comité para debatir acerca del futuro de la ciudad parece facilitar el pluralismo con la idea eje de crear un debate entre los distintos intereses para el bien común de la ciudad.

El proceso de planificación estratégica en Alcobendas pone de manifiesto cómo intereses conflictivos se pueden integrar mediante un proceso de decisión pública. Ninguno de los intereses recibió particular atención. La selección de participantes en el Plan estratégico pone de manifiesto el equilibrio de intereses económicos, las pequeñas y medianas empresas y las grandes, por ejemplo. Tanto la institución como los grupos de interés en la ciudad tienen interés en participar en el Plan Estratégico. Los comerciantes tienen interés en que se mejore el entorno. Para la institución municipal es importante puesto que la inversión empresarial es esencial para la economía de Alcobendas. El Ayuntamiento es el mayor propietario del suelo. Se establece pues una relación de mutuo interés.

\footnotetext{
${ }^{16}$ Entrevistas a los participantes en el proceso de Planificación Estratégica: Empresarios, Sindicatos, Cámara de Comercio y Asociaciones.
} 
En cuanto a la estructura de poder hay un ejercicio del liderazgo por parte de la administración local pero se prioriza el proceso de participación. El Ayuntamiento tiene capacidad para ejercer el liderazgo que exige la elaboración, ejecución e implantación del Plan Estratégico, pero existen otros actores locales con peso en el Plan Estratégico tales como las organizaciones empresariales de la zona norte de Madrid, las grandes empresas localizadas en el territorio municipal, los sindicatos, los comerciantes y las asociaciones de vecinos.

En conclusión el Plan Estratégico de Alcobendas:

1. Se sustenta en un grupo de cargos políticos y gestores públicos que lo impulsan y con la participación de los vecinos y del tejido empresarial local.

2. Los participantes en el proceso son conocedores de la ciudad. El Plan se adecua a las características de la ciudad de Alcobendas.

3. El diseño esta controlado, pero hay participación para definir fines.

4. Es un proceso abierto, se invita a participar a las asociaciones, empresarios, barrios, otras administraciones y a la prensa.

5. Sirve de instrumento educador para los planificadores y para los ciudadanos. En efecto, el Plan Estratégico se constituye en un instrumento de pedagogía para la cooperación ya que hace tomar conciencia a los actores de una solidaridad territorial de tal manera que el propio proceso de elaboración del Plan Estratégico es más importante que los resultados concretos. La Planificación Estratégica en Alcobendas permite la definición de los nuevos objetivos que son compatibles con los particulares de los actores. Asimismo, facilita a los diversos actores el diseño de estrategias comunes e identificar prioridades comunes. El plan tiene un efecto educativo importante: enseña a los participantes acerca de la ciudad. En este sentido es un instrumento para mostrar las relaciones y su posición en la estructura de la ciudad.

6. También se pone el énfasis en el proceso y no en el Plan. Para ello se involucra a los actores del entorno mediante un proceso deliberativo que permite reinterpretar a las elites locales la definición de los problemas públicos locales

7. Finalmente, no es solo un Plan para el desarrollo de la ciudad. El proceso de implicar a un conjunto de actores con intereses en la ciudad para que identifiquen demandas políticas, invitándoles a dibujar el futuro diseño de la ciudad, relegitima la relación de estos con la institución municipal.

\section{CONCLUSIONES}

El proceso de Planificación Estratégica en Alcobendas pone de manifiesto como a pesar de múltiples dificultades de carácter político, organizativo y cultural, una organización pública con una cultura burocrática es capaz de reinventarse a sí misma para redireccionar sus actividades con la finalidad de hacer frente a los retos del entorno para proporcionar mayor bienestar a sus ciudadanos.

Así mismo, evidencia la importancia del liderazgo político y administrativo en los gobiernos locales para la búsqueda del consenso y colaboración entre las élites locales para promover el desarrollo económico y el progreso social, con capacidad para movilizar a los intereses económicos y al tejido asociativo local que son utilizados como inputs en el 
proceso de formulación e implantación de la acción pública local. De este modo, la fuerza motriz de la implantación del Plan Estratégico es una coalición de cargos políticos y directivos que llevan años trabajando juntos y facilita la continuidad del proceso en el tiempo. A ello coadyuvan las mayorías absolutas y mayorías de coalición del partido de gobierno con capacidad y credibilidad para liderar la política de modernización, con el consenso de las fuerzas de la oposición, quienes cuando, posteriormente han alcanzado el poder han seguido los mismos lineamientos modernizadores.

Por otra parte, ha sido clave la responsabilidad de los directivos de área con responsabilidad en la implantación de las distintas líneas estratégicas. Ello ha sido posible por contar el ayuntamiento con una función directiva con personal cualificado en las diversas funciones y la creación en el tiempo de una cultura organizativa en la que la innovación está incentivada. De hecho, las acciones innovadoras son planteadas por los directivos y técnicos, previa a su implantación, dotándoles de un sentido de la participación y haciendo suyas las innovaciones. Del mismo modo el resto de empleados públicos apoyan y se involucran en la implantación de las acciones derivadas del Plan Estratégico en las que ven oportunidades de cualificación y promoción.

Finalmente, y aunque las élites político-administrativas del municipio acotan el conjunto de procedimientos del Plan Estratégico que actúan de marco para la interacción con los intereses económicos y con el tejido cívico, ambos mantienen una actitud favorable durante todo el proceso ya que sus intereses económicos y de participación en las decisiones públicas se han visto favorecidos.

En conjunto, en el proceso de formulación e implantación de la Planificación Estratégica en Alcobendas se ha generado un sistema de interacción, que no es sino un sistema de gobernanza, entre un conjunto de actores (públicos y privados) con diferentes status y variados grados de intereses y estrategias que se introducen en el proceso de decisiones públicas, de tal manera que las decisiones implícitas a la formulación, implantación y evaluación de las políticas y programas públicos locales derivados del proceso de Planificación Estratégica es el resultado de la interacción de diversos actores con interés en la ciudad, configurando así un modelo de gobernanza democrática o manera en que el gobierno local de Alcobendas estructura el ejercicio de la acción pública. Esto no ha significado un desmantelamiento de la estructura administrativa de corte weberiano ni la privatización de los servicios públicos, sino que aquella ha sido complementada con nuevos instrumentos de gestión, consolidando a lo largo del proceso una cultura organizativa enfocada a los resultados y conformando un modelo propio de Gestión Pública.

\section{REFERENCIAS BIBLIOGRÁFICAS}

AGRANOFF, R. (1986): Intergovernmental Management: Human Services Problem Solving in Six Metropolitan Areas, State University of New York Press, Albany, Nueva York.

(2007) - Managing within Networks: Adding Value to Public Organizations, Georgetown University Press, Washington.

ARGYRIS, C., PUTNAM, R. Y MCLAINSMITH, D. (1985): Action Science, Jossey Bass, San Francisco

ARTHUR ANDERSEN (1989): Experiencias en planificación estratégica de ciudades, PROMADRID, Madrid. 
BEHN, R.D. (1993): "Case-Analysis Research and Management Effectiveness: Learning How to Lead Organizations Up Sand Dunes" en Bozeman, B. (coord.), Public Management 40-54, Jossey Bass, San Francisco.

BELTRÁN, M. (2000): "El problema de la calidad en los servicios públicos”, en: Trinidad Requena, A. (coord.), Evaluación y Calidad en las organizaciones públicas, Ministerio de Administraciones Públicas, Madrid.

BRUGUÉ, Q.; GOMÁ, R. Y SUBIRATS, J. (2002): "Gobierno y territorio: del Estado a las redes", en: Subirats, J. (coord.), Redes, territorios y gobierno. Nuevas respuestas locales a los retos de la globalización, Diputació de Barcelona, Barcelona.

BRYSON, J.M. (2004): Strategic Planning for public and nonprofit organizations: A guide to strengthening and sustaining organizational achievement, 3a ed., Jossey Bass, San Francisco.

DENTERS, B. Y ROSE, E. (EDS.) (2005): Comparing Local Governance: Trends and Developments, Palgrave Macmillan, New York

FERNÁNDEZ GÜELL, J.M. (1997): Planificación Estratégica de ciudades, Editorial Gustavo Gili, Barcelona.

FRENCH, P.E. Y FOLZ, D.H. (2004). Executive Behavior and decision making in small U.S. cities. American Review of Public Administration, 34 (1), p. 52-66.

FUNG. A. (2006): Varieties of participation in Complex Governance, Public Administration Review, Diciembre 2006, número especial. p. 35-48.

GONZÁLEZ GÓMEZ, F. Y ORTEGA ALMÓN, M. (2003): Una panorámica sobre el crecimiento de las ciudades: aportaciones más recientes en el campo de la economía, Ciudad y Territorio, Estudios Territoriales, XXXV (135), p. 37-52

KAUFMAN, H. (1960): The Forest Ranger: A Study in Administrative Behavior, H. Hopkins Press, Baltimore.

LARREA ARANGUREN, M. (2003): Clusters y territorio: retos del desarrollo local en la Comunidad Autónoma del País Vasco. Ekonomiaz, 53, p. 138-159

LYNN, L.E. (JR) (1996): Public Management as Art, Science and Profession, Chatam House, Chatam.

MCGREGOR, E.B. (1993): "Toward a Theory of Public Management Success" en Bozeman, B. (coord.) Public Management, Jossey Bass, San Francisco.

MILLER, D. Y MINTZBERG, H. (1983). "The case for configuration" en Beyond Method: Strategies for Social Research, Morgan G., (coord.), Sage, Beverly Hills.

MINTZBERG, H., (2002): La estructuración de las organizaciones, Ariel, Barcelona

MINTZBERG, H., LAMPEL, J., QUINN, J. Y GHOSAL, S. (2003): The strategy process: Concepts, contexts, cases. Prentice Hall. Upper Saddle River.

PASCUAL i ESTEVE, J. (2002): La gestión estratégica de ciudades. Un instrumento para gobernar las ciudades en la era info-global, Dirección General de la Administración Local. Consejería de Gobernación. Junta de Andalucía, Sevilla.

PERRY, J.L. Y KRAEMER, K.L. (1990): "Research Methodology in Public Administration: Issues and Patterns" en Lynn y Wildavsky (eds.), Public Administration: The State of the Discipline, Chatman House, New Jersey.

POISTER, T.H. Y STREIB, G. (2005): Elements of Strategic Planning in management and municipal government: Status after two decades. Public Administration Review, 65 (1) p. 45-56

PORTH, S. (2003): Strategic management: A cross-functional approach, Prentice Hall. Upper Saddle River

STOKER, G. (2008): Mayors and Urban Governance: Developing a Facilitative Leadership Style, Public Administration Review, Julio-Agosto, 2008, p. 722-728.

VILLORIA, M. (1997): “Modernización Administrativa y gobierno postburocrático”, en: Bañón, R. y Carrillo, E. (comps.), La nueva Administración Pública, Alianza Editorial, Madrid

WHEELAND, C. (2003) Implementing a community-wide strategic plan: Rock Hill's empowering vision 10 years later. American Review of Public Administration, 33 (1), p. 460-475 
